\title{
Contaminación biológica intradomiciliaria y su relación con síntomas respiratorios indicativos de asma bronquial en preescolares de Bucaramanga, Colombia
}

\author{
Astrid B. Herrera1', Laura A. Rodríguez¹, Jürg Niederbacher ${ }^{2}$ \\ ${ }^{1}$ Departamento de Salud Pública, Escuela de Medicina, Universidad Industrial de Santander, Bucaramanga, \\ Colombia \\ 2 Departamento de Pediatría, Escuela de Medicina, Universidad Industrial de Santander, Bucaramanga, \\ Colombia.
}

Introducción. La contaminación en el hogar puede desempeñar un papel importante en el desarrollo y exacerbación del asma infantil, sobre todo entre niños genéticamente predispuestos.

Objetivos. Evaluar la asociación entre la presencia de contaminantes biológicos intradomiciliarios y síntomas indicativos de asma, en preescolares de Bucaramanga, Colombia

Materiales y métodos. Se hizo un estudio de corte transversal analítico, en menores de 7 años, en dos zonas de la ciudad con niveles diferentes de contaminación atmosférica por material particulado. Se evaluaron los síntomas respiratorios indicativos de asma y los contaminantes intradomiciliarios mediante cuestionarios previamente validados, las mediciones biológicas de ácaros y hongos por métodos de laboratorio estandarizados, y se usó el modelo log binomial para el análisis multivariado, que permitió evaluar asociaciones mediante la estimación de las razones de prevalencia.

Resultados. Participaron 678 niños, con edad media de 42 meses. La prevalencia de síntomas respiratorios indicativos de asma fue 8,0\% (IC95\%: 5,6-9,6), sin diferencias significativas entre las dos zonas de contaminación extradomiciliaria. El modelo binomial mostró que los síntomas de asma se asociaron con la presencia de: ácaros [razón de prevalencia (RP)=1,78; IC95\%: 1,0-3,0] y Acremonium sp. ( $R P=6,2$; IC95\%: 3,8-10,0), y antecedentes de neumonía ( $R P=4,0 ; I C 95 \%: 2,5-6,4)$, de rinitis alérgica ( $R P=1,9$; IC95\%: 1,25-3,1), de prematuridad ( $R P=3,4 ; \mathrm{IC} 95 \%: 1,7-6,5)$, de padres con asma $(\mathrm{RP}=2,6 ; \mathrm{IC} 95 \%: 1,4-5,0)$ y de tenencia de mascotas (RP=0,4; IC95\%: 0,2-0,9).

Conclusiones. La exposición a contaminantes biológicos intramurales (ácaros y hongos), los antecedentes personales de prematuridad, neumonía o rinitis y el antecedente familiar de asma, incrementan la probabilidad de presentación de síntomas indicativos de asma bronquial en la población estudiada.

Palabras clave: signos y síntomas respiratorios, asma, contaminación del aire, preescolar, Colombia

Biological pollution and its relationship with respiratory symptoms indicative of asthma, Bucaramanga, Colombia

Introduction. Indoor air pollution may play an important role in development and exacerbation of asthma in children.

Objective. The association between the presence of indoor biological contaminants and respiratory symptoms related to asthma was assessed in preschool children.

Materials and methods. This cross-sectional study was undertaken in Bucaramanga, Colombia, and included children $<7$ years of age living in two urban areas of with different levels of outdoor air pollution. The 678 children were an average of 3.5 years of age. Respiratory symptoms indicative of asthma and indoor air pollutants were assessed by previously validated questionnaires.. Biological samples potentially containing mites and fungi were collected by standardized laboratory methods. The log binomial regression model was used for multivariate analysis, using adjusted prevalence ratios (PR).

Results. The prevalence of asthmatic respiratory symptoms was 8.0\%; (95\% C.I: 5.6-9.6), without significant differences between the two areas. Binomial model analysis showed that asthma symptoms were associated with mites (PR 1.78; 95\% C.I. 1.0-3.0), Acremonium sp (PR 6.24; 95 C.I.: 3.8-10.0) and a history of child pneumonia (PR 4.0; 95\% C.I. 2.5-6.4), allergic rhinitis (PR 1.9; 95\% C.I.: 1.23.1), prematurity (PR 3.4; 95\% C.I. 1.8-6.5), parents with asthma (PR 2.6; 95\% C.I. 1.4-5.0) and pet ownership (PR 0.4; 95\% C.I. 0.2-0.9).

Conclusions. The indoor exposure to biological contaminants (dust mites and fungi), history of prematurity, pneumonia, rhinitis and family history of asthma increased the occurence of symptoms suggestive of asthma in young children.

Key words: signs and symptoms, respiratory; asthma; air pollution; Child, preschool; house dust mites, Colombia. 
El asma es considerada uno de los problemas de salud pública más frecuentes e importantes en la mayoría de las grandes ciudades del mundo $(1,2)$. Se ha investigado ampliamente la fisiopatología, la patogénesis, el diagnóstico y el tratamiento farmacológico; sin embargo, en los últimos años la morbilidad relacionada con esta condición se ha incrementado, a pesar de disponer de intervenciones farmacológicas para el control de la enfermedad $(3,4)$.

Este aumento en la prevalencia del asma en los últimos años, ha generado la búsqueda de posibles factores desencadenantes y de las condiciones que favorecen su desarrollo y, entre ellas, los contaminantes ambientales son los que mejor explican el comportamiento de esta enfermedad (5). Los agentes biológicos se encuentran entre los factores ambientales implicados en el desarrollo del asma, incluyendo alérgenos de cucarachas, roedores, ácaros de polvo y hongos (6-8). La estrategia global para el manejo y la prevención del asma, de la Global Initiative for Asthma (GINA) y la Environmental Protection Agency (EPA) $(9,10)$, han documentado que el asma puede ser ocasionada y agudizada por factores ambientales.

Aunque se conocen los efectos de la exposición de la contaminación atmosférica externa medida por material "particulado" (sic.) (particulate matter) y ozono sobre la infección respiratoria aguda, el asma y las enfermedades alérgicas $(11,12)$, existen importantes vacíos en los datos relativos a las exposiciones a largo plazo a concentraciones bajas y a mezclas de diferentes contaminantes.

Bucaramanga cuenta con uno de los indicadores más altos de morbilidad debida a afectaciones de las vías respiratorias, especialmente en población pediátrica de 0 a 4 años de edad, en la que se que presenta una prevalencia de asma entre las más altas del país; así lo refleja el estudio realizado en seis ciudades de Colombia, en el cual la prevalencia de asma fue de 3,5\% en Bogotá, $5,6 \%$ en Barranquilla $7,2 \%$ en Cali, $7,9 \%$ en Medellín, $8,1 \%$ en San Andrés Islas y $8,8 \%$ en Bucaramanga (13).

\section{Correspondencia:}

Astrid Berena Herrera, Carrera 32 N $^{\circ}$ 29-31, Facultad de Salud, Escuela de Salud Pública, Universidad Industrial de Santander, Bucaramanga, Colombia

Teléfono: (315) 6226380

aherrera_lopes@hotmail.com

Recibido: 22/12/10; aceptado:11/05/11
El ambiente intradomiciliario es especialmente relevante porque los niños pasan la mayor parte del tiempo en el interior del hogar (14), y la calidad de dichos ambientes está en relación directa con las características de la vivienda y con el estilo de vida.

Los estudios epidemiológicos demuestran la asociación de los efectos de la contaminación intradomiciliaria, con los síntomas respiratorios y el asma bronquial en niños (15-19). Los resultados derivados de estos estudios, sugieren la hipótesis de que la exposición temprana a los contaminantes en el hogar influye negativamente en la inmunidad innata y adaptativa. También, sugieren que esta alteración inmunológica aumenta el riesgo de desarrollar enfermedades respiratorias causadas por rinovirus y el virus sincitial respiratorio. La concomitancia entre la predisposición genética y el entorno, incrementa el riesgo de enfermedades alérgicas y el asma.

Cada vez es mayor la certeza de que el ambiente de interiores puede desempeñar un papel importante en la patogenia del asma infantil, tanto en su desarrollo como en la exacerbación del asma bronquial, sobre todo entre las personas genéticamente predispuestas (20).

En 2005, empezó en Bucaramanga un proceso de investigación orientado a evaluar la calidad del aire, con el objeto de determinar los efectos de la contaminación atmosférica sobre la salud respiratoria en la población pediátrica. Como resultado de esta investigación, se concluyó que la contaminación atmosférica externa medida por material "particulado" (sic.) (particulate mattter 10, PM10), no mostró relación con los síntomas irritativos oculares y respiratorios en niños menores de siete años, en dos zonas de diferentes niveles de contaminación (21). En consecuencia, se decidió explorar si los contaminantes biológicos (ácaros del polvo doméstico y hongos) y químicos (aerosoles, humo de leña y cigarrillo) en el hogar, tenían un mayor efecto en la presentación de síntomas indicativos de asma en niños menores de siete años.

El objetivo del presente estudio fue determinar la magnitud de la asociación entre la presencia de contaminantes biológicos intradomiciliarios y la presentación de síntomas respiratorios indicativos de asma bronquial en niños menores de siete años, en zonas con diferentes niveles de contaminación atmosférica en Bucaramanga, Colombia. 


\section{Materiales y métodos}

\section{Tipo de estudio, población y muestra}

Se llevó a cabo un estudio analítico de corte transversal entre noviembre de 2009 y marzo 2010, en niños menores de siete años que residían en dos zonas de la ciudad, con diferentes niveles de contaminación atmosférica externa, de acuerdo con los registros de PM10 de la red de vigilancia de la Corporación Autónoma Regional para la Defensa de la Meseta de Bucaramanga.

\section{Tamaño de la muestra}

Se calculó un tamaño de muestra en el subprograma StatCalc del paquete estadístico Epilnfo 6.04d (22), teniendo en cuenta los siguientes parámetros: una significancia estadística de 0,05 , un poder de $80 \%$, una prevalencia esperada de síntomas respiratorios en la zona alta de 22,4; (IC95\%: 18,427,0 ), en la zona baja de 28,9; (IC95\%: 24,5-33,9). Estos resultados se obtuvieron en el estudio previo de contaminación externa, con un riesgo relativo de 1,71 (odds ratio), estimándose una muestra de 632 niños. Además, se calculó un porcentaje de falta de participación de $5 \%$, con lo cual se determinó una muestra de 664 niños.

\section{Selección de los participantes}

Los sujetos se seleccionaron mediante muestreo no probabilístico por conveniencia de los niños que participaron en el proyecto previo de contaminación y que vivieran en las zonas del estudio (23). A partir del centro del lugar donde se encontraba instalado el medidor de material "particulado" (sic.), se establecieron cuatro cuadrantes y se empezó a "barrer" desde el punto del medidor hacia fuera. Se incluyeron niños menores de siete años con residencia de más de 12 meses en el sector y aceptación de participación voluntaria por los padres mediante consentimiento informado por escrito. Se excluyeron los niños con enfermedades cardiacas o neurológicas crónicas.

\section{Variable de respuesta o dependiente}

La variable de desenlace fue la presencia de síntomas respiratorios indicativos de asma, que se midió por los cuestionarios del Estudio Internacional de Sibilancias en Lactantes (EISL) y el International Study of Asthma and Allergies in Childhood (ISAAC) en su versión validada al español $(24,25)$. Para poner en operación esta variable fue necesario utilizar la metodología de Rasch (26), que permitió integrar las preguntas de los dos cuestionarios y establecer un punto de corte con una probabilidad mayor de $50 \%$ en la identificación de niños con síntomas respiratorios indicativos de asma. Se optó por este tipo de análisis, porque permite la medición conjunta de personas e ítems en una misma dimensión y, también, por la falta de una medida de resumen derivada de los cuestionarios EISL e ISAAC.

\section{Exposición de interés}

Los contaminantes biológicos intramurales reco-lectados fueron ácaros y hongos. Para la identificación y cuantificación de la presencia de ácaros se tomaron muestras de polvo con una aspiradora portátil (Vacuum Cleaner Listo $\circledast$, 1.300 watios). El sitio de recolección del muestreo fue el colchón, cubriendo un área aproximada de $2 \mathrm{~m}^{2}$. Las muestras se recolectaron en filtro de tela, adaptado al tubo prolongador de la aspiradora. Además, para evitar la contaminación entre cada toma de las muestras, se limpiaba el tubo aspirador con ácido acético y ácido benzoico. Las muestras se guardaron en bolsas herméticamente cerradas y marcadas con los datos de identificación del participante y la fecha de recolección; luego se almacenaron en una cava de icopor hasta su envío para el análisis.

El contenido de la muestra de polvo recolectado se pasó del filtro a cajas de Petri; después, se pesó en balanza digital y se dispersó el polvo en la caja con ayuda de jeringas de $1 \mathrm{ml}$ para formar cuatro cuadrantes para el análisis del contenido del polvo. Las muestras se revisaron tres veces en busca de ácaros con ayuda de un estereoscopio. Los ácaros vivos y muertos se buscaban cuidadosamente por morfología en cada cuadrante. Por último, cada ácaro se pasó a una lámina que contenía medio Hoyers. Se cubrieron los bordes de la laminilla con acetona comercial y, después de 24 horas, se hizo lectura en el microscopio para identificar la especie de los ácaros. Los ácaros se cuantificaron en cada una de las muestras de polvo doméstico y su cuantificación se expresó como ácaros por gramo de polvo (27).

Las muestras de hongos se recolectaron en cajas de Petri que contenían medio de cultivo sólido (rosa de bengala-cloranfenicol), específico para hongos ambientales. Se ubicaron durante 15 minutos dos cajas de Petri en la cama del niño y en la zona donde jugaba (28); en el caso de menores de un año, las cajas se ubicaron en el corral o en el coche, con supervisión del estudiante de bacteriología.

Las muestras se transportaron al Laboratorio de Micología de la Escuela de Bacteriología de la 
Universidad Industrial de Santander, en cavas de icopor. El tiempo de incubación de las muestras fue de cinco días, por recomendación del laboratorio fabricante. Sin embargo, se hizo seguimiento del crecimiento al tercer y al quinto días.

La medición del peso y la longitud (talla) de los niños, para determinar su estado nutricional, se hizo con los procedimientos estandarizados por la Organización Mundial de la Salud (OMS), usando las tablas de patrones de crecimiento establecidos por la misma organización (29). Las variables sociodemográficas, información general y familiar del niño, exposición a mascotas, características de la vivienda y del dormitorio del niño, se obtuvieron por encuesta estructurada a los padres o cuidadores.

\section{Registro de la información}

La información se recopiló en una agenda digital portátil, en la que se registraban los datos y las mediciones hechas por auxiliares de enfermería entrenadas previamente y bajo la supervisión del coordinador de trabajo de campo.

\section{Procesamiento de la información}

Se verificó el diligenciamiento de la información diariamente en la agenda digital portátil, por parte del grupo de investigadores. El registro de los contaminantes biológicos (ácaros y hongos) se hizo en un archivo Excel® con valores de restricción y validación. Por último, se construyó una base de datos en el programa Excel, para su posterior análisis en el programa Stata $9.0 \circledast$ (30).

\section{Análisis estadístico}

La población se caracterizó por medio de medidas de tendencia central, de posición y de dispersión para las variables continuas y proporciones con IC95\% (31). En el análisis bivariado y estratificado de Mantel y Haenszel, se observó la relación entre la prevalencia de síntomas respiratorios indicativos de asma y los contaminantes intradomiciliarios por medio de la prueba de ji al cuadrado o prueba exacta de Fisher y la t de Student. Finalmente, para establecer la magnitud de las asociaciones entre la variable de respuesta y las variables de estudio, se utilizó un modelo de regresión log - binomial múltiple, para estimar las razones de prevalencia (RP) ajustadas y sus intervalos de confianza del 95 $\%$, siguiendo los criterios de Greeland (32) para la inclusión de las variables en el modelo.

El Comité de Ética en investigación en salud de la Universidad Industrial de Santander, revisó y aprobó el protocolo de investigación.

\section{Resultados}

Se obtuvo información de 748 niños que cumplían los criterios de elegibilidad; sin embargo, 36 (4,83\%) niños cambiaron de domicilio, a 29 (3,89\%) niños no fue posible contactarlos y $5(0,67 \%)$ cuidadores de los niños no permitieron la colocación de la caja de Petri en la habitación del niño y la toma de la muestra de polvo doméstico. Es decir, 648 niños $(87,0 \%)$ fueron captados y se les hicieron mediciones de todas las variables del estudio, con excepción de la muestra de ácaros, que sólo fue posible recoger en 548 muestras de polvo doméstico (figura 1).

Los promedios registrados de material en partículas (PM10) durante el estudio fueron, en la zona de alta contaminación que estaba conformada por los barrios Sanmiguel, La Concordia, Nuevo Sotomayor, Ricaurte y Claverianos, de 58,2 \pm 9,96 $\mu \mathrm{g} / \mathrm{m}^{3}$ (promedio \pm desviación estándar) y, en la zona de baja contaminación que comprendía los barrios La Joya, Pantanos y Alfonso López, de $39,94 \pm 13,9 \mu \mathrm{g} / \mathrm{m}^{3}$.

Las características de la población estudiada se observan en la cuadro 1. El $54 \%$ de los sujetos

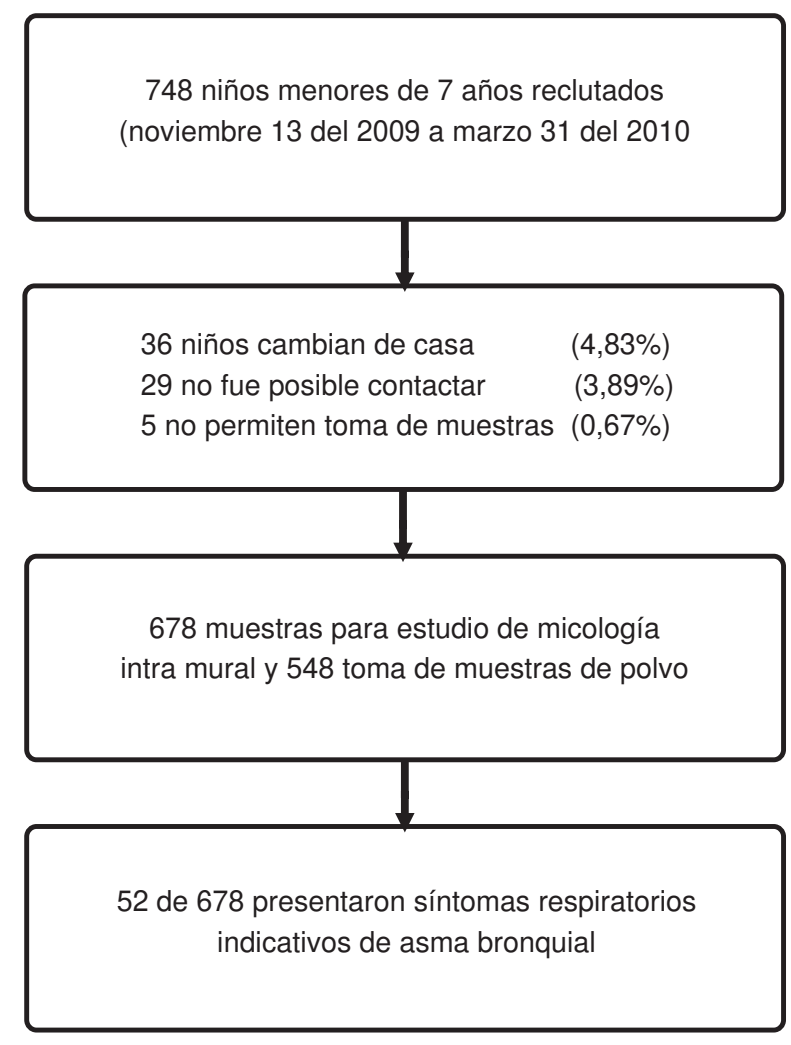

Figura 1. Proceso de inclusión de los participantes en el estudio CIAS. 
Cuadro 1. Características asociadas con la presencia de síntomas respiratorios indicativos de asma bronquial en la población estudiada, Bucaramanga, 2009-2010

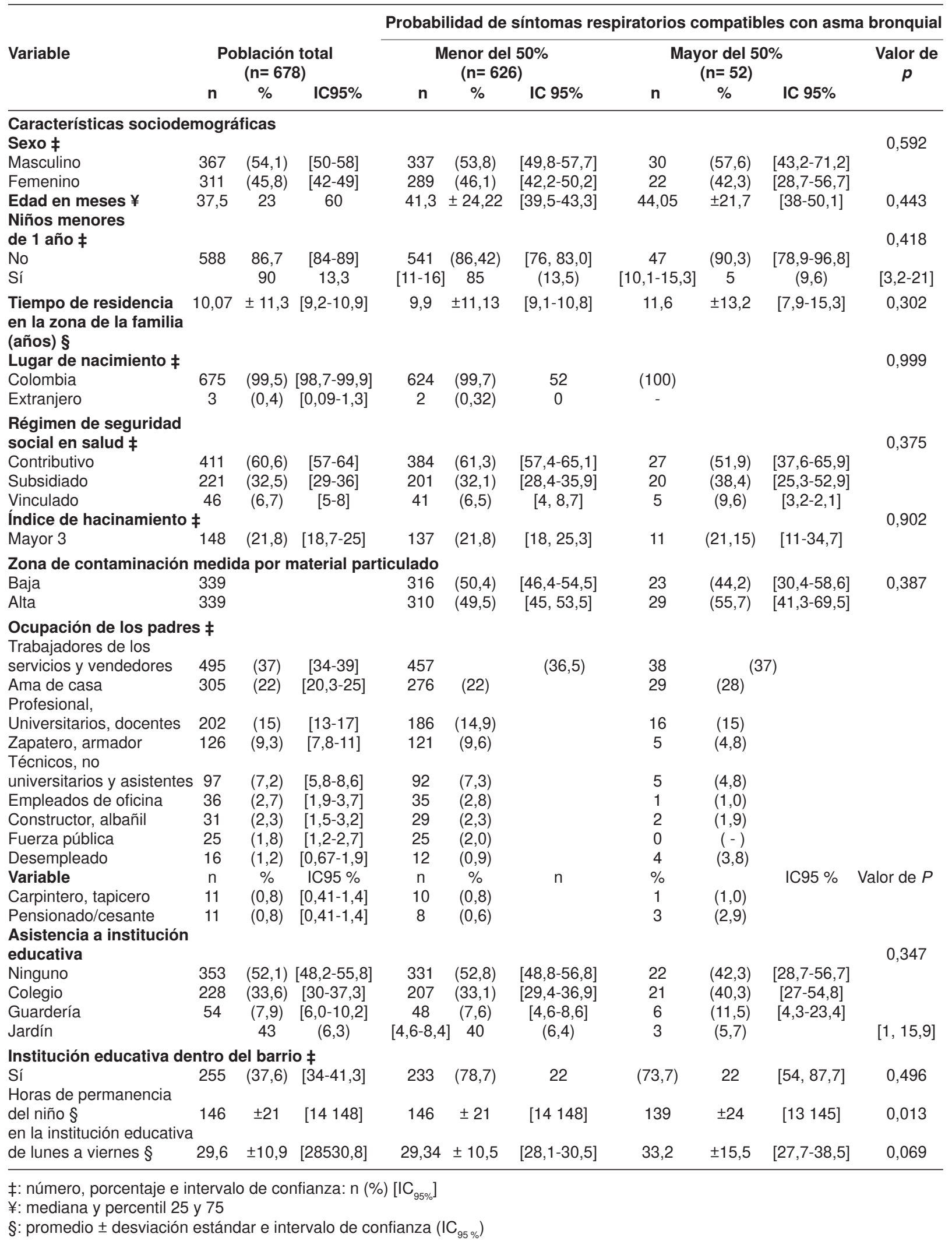


incluidos fueron niños. La mediana de la edad fue 37,5 meses. El $64 \%$ de los niños permanecía bajo el cuidado de la madre. El promedio de horas en casa fue de 146,15 \pm 22 (promedio \pm desviación estándar). El $22 \%$ de los niños vivía en hacinamiento crítico.

La prevalencia de antecedentes personales en los niños fue de $11,3 \%$ para prematuridad y de 12,4 $\%$ para rinitis alérgica; en los participantes no se encontraron antecedentes de tuberculosis. Por el contrario, en los antecedentes patológicos de los familiares, se encontró rinitis en $41 \%$ y asma en $38 \%$. La distribución de la desnutrición aguda, fue: leve, $14,0 \%$; moderada, 3,0 \%, y grave, 3,4 $\%$. El $3,2 \%$ de los niños presentaba sobrepeso (cuadro 2).

El $11,4 \%$ de los familiares fumaban dentro de las viviendas. El 5,6\% de las madres fumó durante el embarazo y el $19,3 \%$ utilizaba aerosoles dentro de la vivienda. Hubo exposición a mascotas en el primer año de vida del niño en el $32 \%$. La tenencia actual de una mascota fue de $39 \%$. La presencia de cucarachas, ratas y ratones dentro la vivienda fue de $28 \%$ y el $6 \%$, respectivamente (cuadro 3 ).

La estructura de las casas de los participantes se caracterizaba por tener paredes frisadas (87 $\%)$, pisos de baldosa (77 \%) y techos de placa (52 \%). Los agentes contaminantes alrededor de la vivienda se distribuyeron así: presencia de zapaterías (34\%), alto flujo vehicular $(24,3 \%)$ y construcciones $(9,4 \%)$.

En el interior de la vivienda de los participantes, se observó la presencia de, al menos, una especie de hongos. La distribución por género fue: Cladosporium sp. (98\%), seguido de Fusarium sp. (82\%), Aspergillussp. (54\%), Penicillium sp. (49\%) y Acremoniumsp. (7,82\%). El promedio de unidades formadoras de colonias (UFC) en el interior de las viviendas fue $40 \pm 2,14 \mathrm{UFC} / \mathrm{m} 3$. Las especies predominantes de ácaros, en concentraciones de polvo con un promedio de 0,35 gramos, fueron Dermatophagoides sp. (16,4 \%), Cheyletus sp. (3,5\%), Lepidoglyphus destructor (2,5\%) y Blomia tropicales $(2,1 \%)$ (cuadro 4). En la zona de alta y la de baja contaminación extradomiciliaria, las concentraciones de polvo doméstico fueron 0,21 y $0,51 \mathrm{~g}$, respectivamente. No hubo diferencias significativas entre los síntomas respiratorios y las concentraciones de polvo doméstico, cuando se compararon en las dos zonas de diferente contaminación extradomiciliaria.
Se estimó una prevalencia de síntomas indicativos de asma bronquial de $8 \%$; (IC95\%: 5,6-9,6), por los cuestionarios ISAAC y EISL. La prevalencia de sibilancias a lo largo de la vida de los participantes fue $29 \%$; (IC95\%: 25-32) para los niños mayores de un año. La prevalencia de sibilancias en los últimos 12 meses fue $23 \%$ (IC95\%: 14-32) en niños menores de un año y fue $17 \%$ (IC95\%: 1420) en niños mayores de un año.

En el análisis bivariado, se observó que las variables que se asociaban con la presencia de síntomas respiratorios indicativos de asma, fueron neumonía, Acremonium sp., ácaros, rinitis alérgica, dermatitis atópica, prematuridad, antecedente familiar de asma y enfermedad pulmonar obstructiva crónica, material del piso y del techo; sin embargo, no hay suficientes observaciones de estas dos últimas variables en las categorías de comparación, para hacer el análisis. Las exposiciones del niño en el hogar a tabaquismo, aerosoles, cucarachas y características de la vivienda, mostraron ausencia de asociación significativa con la presencia de síntomas respiratorios indicativos de asma.

En el cuadro 5 se presenta el modelo de regresión log binomial múltiple y se observan asociaciones estadísticamente significativas para los antecedentes respiratorios de neumonía, rinitis, prematuridad y padres con asma bronquial, y para la presencia del hongo Acremonium sp. (RP=6,2; IC95\%: 3,8-10,0) y de especies de ácaros (RP=1,7; IC95\%:1,0-3,0); estas variables se relacionan con la probabilidad de presentar síntomas respiratorios indicativos de asma. En cuanto a la tenencia de mascotas, se encontró una relación inversa, es decir, es un factor protector con respecto a la presencia de los síntomas respiratorios de asma en la población estudiada.

En el análisis de efectos heterogéneos con el modelo binomial múltiple, se observó que en la zona de alta contaminación la presencia de Acremonium sp. representa una razón de prevalencia de 13,2 (IC95\%: 6,19-28), mientras que, en la zona baja contaminación, una de 2,25 (IC95\%: 0,35-14,4). Además, en la zona de alta contaminación se observó que la neumonía representaba una razón de prevalencia de 5,29 (IC95\%: 2,06-13,6), que fue menor en comparación con la de baja contaminación, que mostró una asociación mayor: razón de prevalencia de 7,1 (IC95\%: 2,8-17,8); la prematuridad mostró una razón de prevalencia de 6,5 (IC95\%: 2,9-14,9), mayor que al compararla con la zona de baja contaminación: razón de 
Cuadro 2. Relación de antecedentes de enfermedades respiratorias en niños y familiares del estudio CIAS, Bucaramanga, $2009-2010$

\begin{tabular}{|c|c|c|c|c|c|c|c|c|c|c|}
\hline \multirow{3}{*}{ Variable } & & & & \multicolumn{7}{|c|}{ Probabilidad de síntomas respiratorios compatibles con asma bronquial } \\
\hline & \multicolumn{3}{|c|}{$\begin{array}{l}\text { Población total } \\
\quad(n=678)\end{array}$} & \multicolumn{3}{|c|}{$\begin{array}{l}\text { Menor del } 50 \% \\
\quad(n=626)\end{array}$} & \multicolumn{3}{|c|}{$\begin{array}{l}\text { Mayor del } 50 \% \\
\quad(n=52)\end{array}$} & \multirow[t]{2}{*}{ Valor de $p$} \\
\hline & $\mathbf{n}$ & $\%$ & IC95\% & $\mathbf{n}$ & $\%$ & IC 95\% & $\mathbf{n}$ & $\%$ & IC 95\% & \\
\hline \multicolumn{11}{|c|}{ Antecedentes patológicos del niño } \\
\hline $\begin{array}{l}\text { Prematuridad } \neq \\
\text { Semanas de gestación }\end{array}$ & 77 & $(11,3)$ & {$[8,9-14]$} & 65 & $(10,3)$ & {$[7,9-12,7]$} & 12 & $(23,1)$ & {$[11,2-34,9]$} & 0,006 \\
\hline en prematuros $\S$ & 33,1 & $\pm 2,8$ & {$[32,4-33,7]$} & 33,5 & $\pm 2,5$ & {$[32,8-34,2]$} & 31,1 & $\pm 3,6$ & {$[28,7-33,4]$} & 0,059 \\
\hline \multicolumn{11}{|c|}{ Enfermedades respiratorias } \\
\hline Rinitis alérgica $\ddagger$ & 84 & $(12,4)$ & {$[9-15]$} & 71 & $(11,3)$ & 13 & (25) & & 0,004 & \\
\hline Asma $\ddagger$ & 43 & $(6,3)$ & {$[4,5-8,1]$} & 21 & $(3,3)$ & & 22 & $(42,3)$ & 0,000 & \\
\hline Neumonía ‡ & 32 & $(4,7)$ & {$[3-6,3]$} & 23 & $(3,6)$ & {$[2,2-5,1]$} & 9 & $(17,3)$ & {$[6,7-28]$} & 0,000 \\
\hline $\begin{array}{l}\text { Dermatitis atópica } ¥ \\
\text { Síndrome }\end{array}$ & 23 & $(3,3)$ & {$[2-4,7]$} & 19 & $(3,0)$ & {$[1,7-4,4]$} & 4 & $(7,6)$ & {$[2,0-15]$} & 0,075 \\
\hline broncoobstructivo) $\ddagger$ & 15 & $(2,2)$ & {$[1-3]$} & 11 & $(1,7)$ & {$[0,7-2,8]$} & 4 & $(7,6)$ & {$[0,2-15,2]$} & 0,005 \\
\hline Peso $(\mathrm{kg}) \S$ & 15,6 & $\pm 6,5$ & {$[15,1-16,1]$} & 15,5 & $\pm 6,7$ & {$[15,0-16,1]$} & 15,7 & $\pm 5,1$ & {$[14,3-17,2]$} & 0,798 \\
\hline Talla $(\mathrm{cm}) \S$ & 96,2 & $\pm 18,7$ & [95-98] & 96,1 & $\pm 18,8$ & {$[94,5-97,5]$} & 96,2 & $\pm 18,7$ & {$[94,8-97,6]$} & 0,416 \\
\hline Estado nutricional $¥$ & & & & & & & & & & 0,608 \\
\hline Normal & 541 & $(79,7)$ & [76-83] & 502 & $(80,2)$ & {$[70,5-77,3]$} & 39 & $(75)$ & {$[4,1-7,7]$} & \\
\hline Leve & 92 & $(13,6)$ & {$[11-16]$} & 82 & $(13,1)$ & {$[9,7-14,7]$} & 10 & $(19,2)$ & {$[0,7-2,7]$} & \\
\hline Moderado & 22 & $(3,2)$ & {$[2,0-4,8]$} & 21 & $(3,3)$ & {$[1,9-4,7]$} & 1 & $(1,9)$ & {$[0,0-0,8]$} & \\
\hline Grave & 23 & $(3,4)$ & {$[2,2-5]$} & 21 & $(3,3)$ & {$[1,9-4,7]$} & 2 & $(3,8)$ & {$[0,0-1,0]$} & \\
\hline \multicolumn{11}{|c|}{ Familiares con antecedentes patológicos de: } \\
\hline Rinitis alérgicał & 277 & $(40,8)$ & [37-44] & 252 & $(40,2)$ & [36-44] & 25 & $(48,1)$ & [34 -62] & 0,270 \\
\hline Asma $\ddagger$ & 259 & $(38,2)$ & [34-41] & 229 & $(36,5)$ & [32-40] & 30 & $(57,7)$ & {$[44-71]$} & 0,003 \\
\hline EPOC $\neq$ & 30 & $(4,4)$ & {$[2,8-5,9]$} & 25 & $(3,9)$ & {$[2,4-5,5]$} & 5 & $(9,6)$ & {$[1,3-17]$} & 0,058 \\
\hline Bronquitis ₹ & 56 & $(8,3)$ & [6-10] & 51 & $(8,1)$ & {$[6-10,3]$} & 15 & $(9,62)$ & {$[1,3-17,3]$} & 0,712 \\
\hline Neumonía ‡ & 53 & $(7,8)$ & {$[6-9,8]$} & 47 & $(7,5)$ & {$[5,4-9,6]$} & 6 & $(11,5)$ & {$[2,5-20,5]$} & 0,298 \\
\hline Tuberculosis ¥ & 31 & $(4,5)$ & [2,9-6] & 28 & $(4,4)$ & {$[2,8-6,0]$} & 3 & $(5,77)$ & {$[-0,78-12,3]$} & 0,667 \\
\hline Dermatitis atópica $\ddagger$ & 25 & $(3,6)$ & {$[2-5,1]$} & 23 & $(3,6)$ & {$[2,2-5,2]$} & 2 & $(3,85)$ & {$[-, 1,6-9,2]$} & 0,950 \\
\hline
\end{tabular}

prevalencia de 1,72 (IC95\%: 0,65-4,56). Se debe resaltar que la contaminación atmosférica externa no explica la presentación del asma.

Al analizar los cuestionarios EISL e ISAAC con la metodología de Rasch, se encontró un buen nivel de ajuste al modelo, tanto para los sujetos como para los ítems, por lo que se puede afirmar que estos instrumentos miden los síntomas respiratorios indicativos de asma, siendo estos cuestionarios unidimensionales.

\section{Discusión}

En el presente estudio se encontraron asociaciones estadísticamente significativas entre la presencia de contaminantes biológicos (Acremonium sp. y los ácaros), los antecedentes respiratorios personales y familiares, y la presentación de síntomas respiratorios indicativos de asma bronquial. Es decir, estas variables aumentan la probabilidad de presentación del asma. La presencia de mascotas en el hogar tiene una relación inversa: es un factor protector contra la presencia de síntomas indicativos de asma en la población estudiada.

Otros resultados de estudios epidemiológicos (33-36) han estimado asociaciones entre los géneros fúngicos Cladosporium sp., Alternaria sp., Aspergillus sp., Acremonium sp., Absidia sp. y Penicillum sp., con los síntomas respiratorios de asma, rinitis y otras enfermedades alérgicas. Estos trabajos, además, han permitido identificar los hongos alérgenos más importantes y la agudización de la sintomatología en personas con asma y rinitis alérgica. Sin embargo, en la literatura científica existen pocos estudios que relacionen el Acremonium sp. y la sintomatología asmática, como se estableció en este trabajo.

Por otra parte, la EPA ha documentado que las especies de hongos prevalentes en el interior de la casa en estudios en Estados Unidos, y corresponden a Aspergillus sp., Penicillium sp., 
Cuadro 3. Contaminantes en el interior de la vivienda de los participantes del estudio CIAS, Bucaramanga, 2009 - 2010

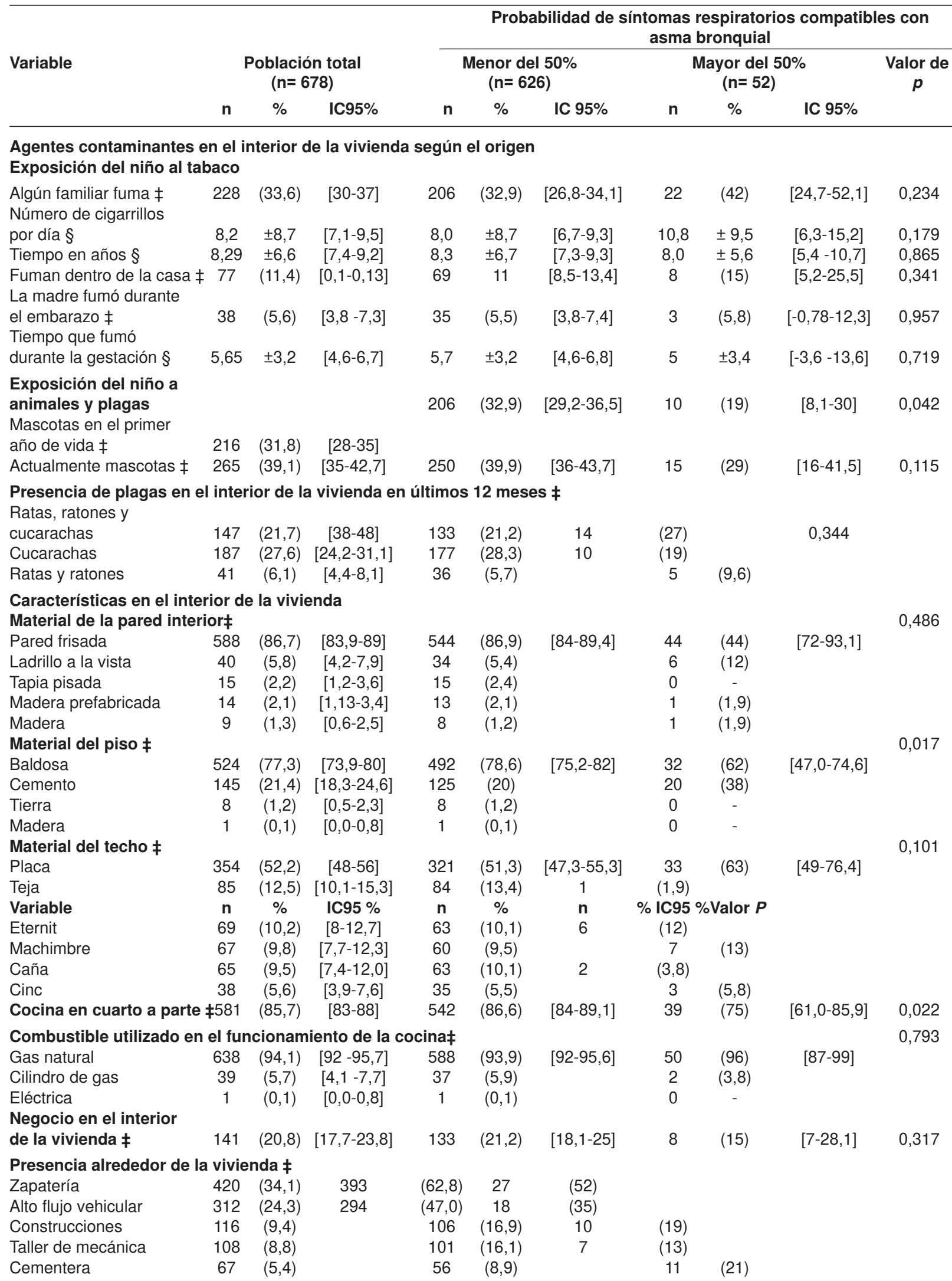


Biomédica 2011;31:357-71

Pinturas
Ornamentación
Ferretería
Basureros
Tapicería
Utilización de aerosoles
en el interior de
la vivienda $¥$
Tipo de aerosol $¥$
Insecticida
Ambientador
Limpieza
Pintura

$\begin{array}{ll}60 & (4,8) \\ 56 & (4,5) \\ 41 & (3,3) \\ 37 & (3,0) \\ 32 & (2,6)\end{array}$

Reformas en la casa en los

Sí

Habitación del niño

Sala

Baño

Cocina

Pintaron la habitación

del niño en los últimos

12 meses $\ddagger$

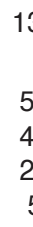

$\begin{array}{ccc}131 & (19,3) & {[16,4-22,5]} \\ 58 & (44,2) & {[35,6-53,2]} \\ 43 & (32,8) & {[24,8-41,5]} \\ 25 & (19,1) & {[12,7-26,8]} \\ 5 & (3,8) & {[1,2-8,7]}\end{array}$

súltimos 12 meses $¥$

$86 \quad(12,7) \quad[10-15]$

$33 \quad(38,4) \quad 31$

$22 \quad(25,6) \quad 22$

$16 \quad(18,6) \quad 13$

$15 \quad(17,4$

$160 \quad(23,6) \quad[20-26,8]$

Contaminación biológica intradomiciliaria y síntomas respiratorios

\section{7}

52

36

37

31

122

55

38

24

5

80

$(4,9)$

$(3,5)$

$(2,1)$

14
$(9,1)$

$(8,3)$

$(5,7)$

$(5,9)$

$(4,9)$

$(8,7)$

$(6,1)$

$(3,8)$

$(0,8)$

$(12,8) \quad[10,2-15,6]$

$(2,2)$

146
2

0

3

$[16,4-22,8]$

6

$(3,8)$

$(5,8)$

1
$(5,8)$

$(7,7)$

$(9,6)$

$(1,9)$

(17)

$[8,2-30,3]$

0,702

0,480

$(5,8) \quad[1,2-16]$

$(9,6)$

$(1,9)$

0,796

(12) $\quad[4,3-23,4]$

$(1,9)$
Fusarium sp. y Acremonium sp. Asimismo, plantea que las especies Alternaria sp. y Cladosporium $\mathrm{sp}$. se relacionan con la gravedad de los síntomas de asma y su exacerbación en pacientes con asma (37). De la misma manera, en el estudio de contaminación del aire por microorganismos en la localidad de Puente Aranda en Bogotá, se identificaron especies frecuentes de Aspergillus sp. y Penicillum sp., y con menor presencia, Acremonium sp. (38). Estos hallazgos demuestran la similitud con las especies de hongos detectadas en el presente estudio.

Aunque falta consenso en determinar la concentración umbral de esporas alérgenas cuantificadas por unidades formadoras de colonias (UFC/m³) (39-41) en la presentación de síntomas respiratorios, se ha estimado que las concentraciones con un rango entre 100 y $950 \mathrm{UFC} / \mathrm{m}^{3}$ de los géneros Penicillum sp., Aspergillus sp. y Cladosporium sp., incrementan la probabilidad de presentar tos seca y sibilancias en niños menores de 14 años (42). Sin embargo, Kowalski reporta que las UFC de Aspergillus sp. superiores a 50 , se relacionan con tos e irritación de ojos y la piel (43).

En este trabajo no se encontró asociación con la cuantificación de las unidades formadoras de colonias de hongos y los síntomas indicativos de asma; esto podría deberse a que el promedio estimado fue de $40 \mathrm{UFC} / \mathrm{m}^{3}$, el cual se considera bajo al compararlo con el de otros estudios anteriormente mencionados. Asimismo, Pulimood, et al. (44), establecieron que los hongos son causantes de alergias en personas sensibilizadas, pero no siempre se pudo establecer la relación entre la presencia de sintomatología alérgica y el recuento de unidades formadoras de hongos atmosféricos.

Sólo en unos pocos estudios de los que han incluido medidas objetivas de la exposición al moho por cultivo y el conteo de UFC en el interior de las casas, se ha demostrado una asociación positiva entre la exposición y el asma o los síntomas similares a los del asma (45).

Otro factor asociado positivamente con la presencia de síntomas indicativos de asma, fue la presencia de ácaros. Se ha demostrado una fuerte asociación de los ácaros del polvo doméstico con el desarrollo, la gravedad y la morbilidad del asma (46-48). Existe una relación entre la dosis y la respuesta de la exposición a los ácaros, y la presencia de síntomas respiratorios indicativos de asma, tanto en estudios de corte transversal como en estudios prospectivos; la concentración de polvo doméstico estimada en estos estudios ha estado entre 10 y $23,4 \mathrm{mg}$ de alérgenos de $D$. farinae por gramo de polvo. (49). Sin embargo, otros estudios han reportado niveles de alérgenos de $D$. farinae y $D$. pteronyssinus de $2 \mu \mathrm{g} / \mathrm{g}$ de polvo, identificados como factores de riesgo en la sensibilización en personas sensibles; los niveles superiores a $10 \mu \mathrm{g} / \mathrm{g}$ de polvo, se han asociado con la crisis asmática (50).

Con todo lo anterior, al revisar la literatura científica actual, se percibe que los ácaros del polvo doméstico son los alérgenos más frecuentemente relacionados 
Cuadro 4. Distribución de los contaminantes biológicos intramurales en la población estudiada, Bucaramanga, 2009 - 2010

\begin{tabular}{|c|c|c|c|c|c|c|c|c|c|c|}
\hline \multirow{3}{*}{ Variable } & \multirow{2}{*}{\multicolumn{3}{|c|}{$\begin{array}{l}\text { Población total } \\
\quad(n=678)\end{array}$}} & \multicolumn{7}{|c|}{ Probabilidad de síntomas respiratorios compatibles con asma bronquial } \\
\hline & & & & \multicolumn{3}{|c|}{$\begin{array}{l}\text { Menor del } 50 \% \\
\quad(n=626)\end{array}$} & \multicolumn{3}{|c|}{$\begin{array}{l}\text { Mayor del } 50 \% \\
\quad(n=52)\end{array}$} & \multirow[t]{2}{*}{$\begin{array}{l}\text { Valor } \\
\text { de } p\end{array}$} \\
\hline & $\mathbf{n}$ & $\%$ & IC95\% & $\mathbf{n}$ & $\%$ & IC $95 \%$ & $\mathbf{n}$ & $\%$ & IC $95 \%$ & \\
\hline \multicolumn{11}{|c|}{ Exposición a contaminantes biológicos del aire intradomiciliario } \\
\hline $\begin{array}{l}\text { Hora de la toma de } \\
\text { la muestras }\end{array}$ & & & & & & & & & & 0,149 \\
\hline Mañana & 428 & $(63,1)$ & {$[33-40,6]$} & 400 & $(63,9)$ & {$[60-68]$} & 28 & $(54)$ & {$[39,5-68]$} & \\
\hline Tarde & 250 & $(36,8)$ & {$[33-40,5]$} & 226 & $(36,1)$ & {$[32,3-40]$} & 24 & $(46)$ & {$[32,2-60,5]$} & \\
\hline $\begin{array}{l}\text { Unidades formadoras de } \\
\text { colonias en la casa } ¥\end{array}$ & 31 & 20 & 48 & & 19 & 24 & 38,5 & 23 & 55,5 & 0,429 \\
\hline $\begin{array}{l}\text { Unidades formadoras de } \\
\text { colonias en la habitación } ¥\end{array}$ & 11 & 7 & 18 & 11 & 7 & 18 & 12,5 & 8,5 & 19,5 & 0,157 \\
\hline $\begin{array}{l}\text { Unidades formadoras de } \\
\text { colonias en otra habitación }\end{array}$ & & 7 & 17 & 11 & 7 & 17 & 15 & 10 & 23 & 0,525 \\
\hline $\begin{array}{l}\text { Unidades formadoras de } \\
\text { colonias en la sala } ¥\end{array}$ & 15 & 11 & 23 & 15 & 11 & 23 & 20 & 12 & 30,5 & 0,700 \\
\hline $\begin{array}{l}\text { Presencia de hongos } \\
\text { en la casa }\end{array}$ & 678 & & & 626 & 100 & & 52 & 100 & & \\
\hline \multicolumn{11}{|c|}{ Presencia de UFC de hongos por género } \\
\hline Cladosporium sp. & 665 & $(98,0)$ & [97 - 99] & 616 & $(98,4)$ & {$[97,4-99,3]$} & 49 & $(94)$ & {$[87,6-100]$} & 0,035 \\
\hline Fusarium sp. & 557 & $(82,1)$ & [79-85] & 516 & $(82,4)$ & {$[79,4-85,4]$} & 41 & (79) & {$[67,3-90,3]$} & 0,517 \\
\hline Levadura & 458 & $(67,5)$ & [64 -71] & 426 & $(68,1)$ & {$[64,3-72]$} & 32 & (62) & {$[48-75,2]$} & 0,335 \\
\hline Scopulariopsis sp. & 441 & $(65,0)$ & {$[43-53,7]$} & 407 & (65) & {$[61,3-68,7]$} & 34 & (65) & {$[52,0-79]$} & 0,957 \\
\hline Aspergillus sp. & 365 & $(53,8)$ & {$[49-57]$} & 334 & $(53,4)$ & {$[49,4-57]$} & 30 & (30) & {$[43,8-72]$} & 0,547 \\
\hline Penicillium sp. & 334 & $(49,2)$ & [45 - 53] & 304 & $(48,6)$ & {$[44,6-52,4]$} & 30 & (58) & {$[43,8-71,5]$} & 0,206 \\
\hline Absidia sp. & 212 & $(31,3)$ & {$[27-34,7]$} & 194 & (31) & {$[27,3-3,5]$} & 18 & (35) & {$[21,2-48]$} & 0,588 \\
\hline Mucor sp. & 146 & $(21,5)$ & {$[18-24,6]$} & 132 & $(21,1)$ & {$[18-25]$} & 14 & (27) & {$[14,4-39,3]$} & 0,325 \\
\hline Curvularia sp. & 109 & $(16,1)$ & {$[13-18]$} & 102 & $(16,3)$ & {$[13,4-19,2]$} & 7 & (13) & {$[3,9-23]$} & 0,593 \\
\hline Epicoccum sp. & 107 & $(15,7)$ & {$[13-18,5]$} & 102 & $(16,3)$ & {$[13,4-19,2]$} & 5 & $(9,6)$ & {$[1,3-18]$} & 0,204 \\
\hline Acremonium sp. & 53 & $(7,82)$ & {$[5,7-9,8]$} & 44 & $(7,0)$ & {$[5,02-9]$} & 9 & (17) & {$[6,6-2,8]$} & 0,008 \\
\hline Alternaria sp. & 8 & $(1,2)$ & {$[0,3-1,9]$} & 7 & $(1,1)$ & {$[0,3-1,9]$} & 1 & $(1,9)$ & {$[-1,9-5,8]$} & 0,606 \\
\hline Polvo (gramos)§ & 0,35 & $\pm 0,85$ & {$[28-42,7]$} & 0,36 & $\pm 0,88$ & {$[0,2-0,4]$} & 0,21 & $\pm 0,23$ & {$[0,1-28,3]$} & 0,241 \\
\hline $\begin{array}{l}\text { Presencia de ácaros } \\
\text { en el colchón }\end{array}$ & 162 & $(29,5)$ & {$[25,7-33,4]$} & 149 & $(23,8)$ & {$[25,5-33,5]$} & 13 & (25) & {$[16-44,5]$} & 0,920 \\
\hline Dermatophagoides spp. & 111 & $(16,4)$ & {$[13,5-19]$} & 104 & $(16,6)$ & {$[14-19,5]$} & 7 & (13) & {$[3,8-23,0]$} & 0,555 \\
\hline Cheyletus spp. & 24 & $(3,5)$ & {$[2,1-4,9]$} & 20 & $(3,2)$ & {$[1,8-4,6]$} & 4 & $(7,7)$ & {$[0,2-15,2]$} & 0,092 \\
\hline Lepidoglyphus destructor & 17 & $(2,5)$ & {$[0,0-0,03$} & 17 & $(2,7)$ & {$[1,4-3,9]$} & 0 & - & - & 0,229 \\
\hline Blomia tropicales sp. & 14 & $(2,1)$ & {$[0,9-3,1]$} & 14 & $(2,2)$ & {$[1,07-3,4]$} & - & & & 0,276 \\
\hline
\end{tabular}

con el desarrollo de enfermedades alérgicas, como el asma, la rinitis y la dermatitis (51).

Otro factor asociado positivamente con la presencia de síntomas asmáticos, fueron los antecedentes respiratorios de los niños (52), puesto que contribuyen al deterioro de la función pulmonar, haciéndolos aún más sensibles a contaminantes intradomiciliarios. Asimismo, Ngoc, et al. (53), encontraron asociaciones significativas entre la hiperreacción bronquial y el antecedente de asma en los padres y el reporte de moho en el hogar.

Boulet (54) concluyó que las infecciones respiratorias pueden actuar sinérgicamente con alérgenos y exposiciones a contaminantes biológicos, en el desarrollo y la exacerbación del asma, y que la identificación de enfermedades concomitantes es reconocida como una parte integral para la detección de niños con probabilidad de desarrollar asma. Estos hallazgos son similares a los reportados por otros estudios previos $(55,56)$, que han establecido que los antecedentes de enfermedades respiratorias, las sibilancias en la infancia, la sensibilización alérgica y la hiperreacción bronquial, incrementan el riesgo de aparición de tos, sibilancias y asma en la edad adulta.

Existe controversia en relación con la asociación entre la tenencia de mascotas y la presencia de 
Cuadro 5. Variables asociadas con síntomas respiratorios indicativos de asma bronquial en preescolares, regresión binomial múltiple, razonen de prevalencia ajustadas para el modelo final*

\begin{tabular}{lccc}
\hline Variable & \multicolumn{3}{c}{$\begin{array}{c}\text { Presencia de síntomas respiratorios } \\
\text { indicativos de asma bronquial con } \\
\text { una probabilidad de } 50 \%\end{array}$} \\
& RP & IC95\% & $\boldsymbol{P}$ \\
\hline Acremonium sp. & 6,2 & {$[3,8-10]$} & 0,000 \\
Neumonía & 4,0 & {$[2,5-6,4]$} & 0,000 \\
Rinitis alérgica & 1,9 & {$[1,1-3,1]$} & 0,011 \\
Prematuridad & 3,4 & {$[1,7-6,5]$} & 0,000 \\
Padres con asma & 2,6 & {$[1,4-5]$} & 0,003 \\
Ácaros & 1,7 & {$[1,0-3]$} & 0,033 \\
Tenencia de mascotas & 0,4 & {$[0,2-0,9]$} & 0,031 \\
\hline
\end{tabular}

RP: razón de prevalencia

IC95\%: intervalo de confianza de $95 \%$ $\mathrm{p}$ : valor de $p$

síntomas respiratorios indicativos de asma (57). Los hallazgos de la relación entre la exposición a perros o gatos y la sintomatología respiratoria, no son concluyentes $(58,59)$. Sin embargo, en un metanálisis de Takkouche, et al. (60), que incluyó 32 estudios prospectivos, se estimó una medida de asociación resumen (OR) y se encontraron como factores de riesgo la tenencia de perro $(\mathrm{OR}=1,14$; IC95\%: 1,1-1,29) y la exposición a cualquier mascota (OR=1,39; IC95\%: 1,0-1,95) para la presencia de asma. Al contrario de la exposición a perros, la exposición a los gatos ejerce un efecto preventivo contra el desarrollo de asma $(\mathrm{OR}=0,72$; IC95\%: 0,55-0,93); la exposición a mascotas con pelaje no es concluyente en el desarrollo de asma. Estos mismos autores mencionan que los resultados deben interpretarse con precaución debido al pequeño número de estudios realizados en países con baja prevalencia en la tenencia de mascotas $y$, también, porque algunas mascotas producen más alérgeno y endotoxinas bacterianas que otros (61).

En este estudio, la prevalencia actual de tenencia de mascotas en niños con síntomas indicativos de asma fue de $28,25 \% \mathrm{y}$, en el primer año de vida, fue de $19,23 \%$. La prevalencia de tenencia de mascotas en el hogar en nuestro estudio, es baja comparada con la obtenida en el estudio multicéntrico de García, et al. (62), que registró en Colombia una prevalencia de $40,7 \%$ de mascotas en el hogar. En relación con la presencia de mascotas en el hogar, el riesgo fue de 1,18; $\left(\mathrm{IC}_{95 \%}\right.$; $0,91-1,53)$ para tener sibilancias recurrentes en este mismo estudio.
Otro estudio que analizó la exposición a mascotas fue el SIDRIA II, de Lombardi, et al. (63), en 11 ciudades de Italia; sus resultados mostraron que la tenencia de gatos en el primer año de vida indica una probabilidad 1,88 veces mayor (IC95\%:1,33$2,68)$ de presentar sibilancias y 1,74 veces mayor (IC95\%: 1,10-2,78) de desarrollar asma. Sin embargo, no se encontró asociación significativa entre la exposición al perro en el primer año de vida o cualquier otro periodo, y los síntomas indicativos de asma.

En estudios previos se ha establecido el efecto de la edad (64), el sexo, el tabaquismo pasivo (65), la tenencia de una mascota en el primer año de vida y el estado nutricional (66), en la presentación de sibilancias recurrentes y asma; sin embargo, en el presente estudio estos factores de riesgo no explican la presentación de síntomas respiratorios indicativos de asma.

Esimportanteaclararque en este estudio no se utilizó el diagnóstico clínico de asma como desenlace. En su lugar se utilizó una medición indirecta de síntomas respiratorios indicativos de asma, con los cuestionarios EISL e ISAAC $(67,68)$, instrumentos que se han utilizado en estudios previos para estimar la prevalencia de asma, enfermedades alérgicas y la presencia de síntomas respiratorios, con una adecuada reproducibilidad y validez de criterio. Sin embargo, esta medición podría ser más sólida si se utilizaran como complemento pruebas funcionales o inmunológicas que permitieran hacer el diagnóstico clínico de asma.

Una debilidad de este estudio es que las mediciones biológicas se hicieron en tiempo seco y no abarcaron temporadas climáticas diferentes, para establecer de una manera confiable el comportamiento estacional de los hongos y ácaros. Se recolectaron muestras de polvo doméstico del colchón de la cama, pero se podría optimizar la cantidad de polvo recolectada ampliando el muestreo a otros sitios, como almohadas y cortinas.

Un potencial sesgo de información fue el uso de aspiradoras de baja potencia para la recolección de los ácaros en los muestreos iniciales. Posteriormente, se modificó la técnica de recolección y se utilizaron aspiradoras con mayor fuerza; por tal razón, para no incurrir en un sesgo de medición diferencial, se excluyeron las muestras iniciales en el análisis de la información.

Asimismo, se consideró la presencia de sesgo de memoria al responder el instrumento, en el 
sentido de que la pregunta se formulaba tiempo después de presentarse los síntomas indicativos de asma; sin embargo, en caso de existir, este sesgo no fue diferencial entre los participantes, pues se implementó la aplicación del instrumento de la misma forma a todos los participantes por encuestadoras entrenadas.

Entre las fortalezas del estudio puede mencionarse la utilización de la metodología de Rasch para la definición operativa de la variable dependiente, que es una medición objetiva que permite conocer la probabilidad de la respuesta de una escala, teniendo en cuenta la habilidad de la persona y la dificultad del ítem (69), y proporciona una variable de salida unidimensional. De la misma forma, la aplicación de la regresión binomial permite estimar una magnitud más real de la asociación, pues el modelo de regresión logística sobrestima la asociación cuando las prevalencias de los eventos son poco frecuentes o sobrepasan el $10 \%$ de lo observado $(70,71)$.

Se considera otra fortaleza el hecho de que en nuestro medio se han llevado a cabo pocos estudios que exploren la relación entre los síntomas respiratorios de asma y los contaminantes biológicos intramurales (72). Estos hallazgos son importantes para el desarrollo de futuras medidas de prevención e intervención en poblaciones similares a la del estudio.

Esta investigación refuerza la hipótesis de que los síntomas respiratorios indicativos de asma están relacionados con la contaminación biológica en el interior de la vivienda; sin embargo, para próximos estudios se recomienda medir las concentraciones de material en partículas en el interior de las viviendas, y utilizar equipos volumétricos y de compactación para medir con mayor precisión la presencia de hongos.

Por otra parte, los resultados de la presente investigación contribuyen a proponer intervenciones eficaces en el control de contaminantes biológicos en el hogar (73), e identificar tempranamente los niños con antecedentes personales y familiares de enfermedades infecciosa y crónicas, que son factores que predisponen al desarrollo de asma.

En síntesis, la presencia de los factores de riesgo de antecedentes respiratorios personales y familiares, y los contaminantes biológicos (hongos y ácaros) en el hogar, aumenta la probabilidad de tener síntomas indicativos de asma bronquial. Los niveles de contaminación atmosférica externa por
PM 10, no son un factor de riesgo para el desarrollo de síntomas indicativos de asma en la población de estudio. Sin embargo, en los niños que viven en la zona de alta contaminación atmosférica externa, la presencia de Acremonium sp. y la prematuridad tienen un mayor efecto, mientras que, en los que viven en la zona baja, lo tiene el antecedente de neumonía.

Además, la exposición en el hogar a plagas e insectos, los aerosoles y el tabaquismo, son factores no asociados con el desarrollo del evento en la población de estudio. Lo anterior sugiere que, en Bucaramanga, desde edades tempranas hay factores relacionados (ácaros y hongos) con el desarrollo de síntomas indicativos de asma bronquial.

Finalmente, la recomendación es diseñar y evaluar intervenciones preventivas en la eliminación de contaminantes biológicos en el hogar, en especial, en niños con antecedentes de enfermedades respiratorias infecciosas y alérgicas, pues los factores genéticos son más complejos de modificar que el componente ambiental, el cual se presta para analizar y modificar exposiciones de riesgo.

\section{Agradecimientos}

Los investigadores agradecen a los niños participantes y a sus padres. A Luis Carlos Orozco, docente y epidemiólogo de la Universidad Industrial de Santander, por su colaboración y enseñanzas en el análisis con el método de Rasch. A Gustavo Cuadros, docente de la Universidad de La Salle, presidente electo de la Asociación Colombiana de Alergia, Asma e Inmunología, e investigador en alergia humana, por el procesamiento de las muestras de polvo doméstico y la identificación de los ácaros. A la Compañía Nacional de Chocolates, S. A., por el apoyo logístico en la divulgación de los resultados de este estudio en la comunidad.

\section{Conflicto de intereses}

Los autores declaran que no poseen intereses comerciales o económicos sobre los resultados presentados del estudio.

\section{Financiación}

Este proyecto fue financiado por la convocatoria interna de la Vicerrectoría de la Investigación y Extensión de la Universidad Industrial de Santander, y se inscribió con el código 5653 en la Dirección de Investigación y Extensión de la Facultad de Salud de la misma. Otras entidades financiadoras fueron el Observatorio de Salud 
Pública de Santander y la Secretaría de Salud y Ambiente de Bucaramanga.

\section{Referencias}

1. Cooper P, Rodríguez LC, Cruz AA, Barreto ML. Asthma in Latin America: A public heath challenge and research Opportunity. Allergy. 2009;64:5-17.

2. Mullol J, Valero A, Alobid I, Bartra J, Navarro AM, Chivato $\mathrm{T}$, et al. Allergic rhinitis and its impact on asthma update (ARIA 2008). The perspective from Spain. J Investig Allergol Clin Immunol. 2008;18;327-34.

3. Rosales JA, Torres VM, Olaiz G, Borja VH. Los efectos agudos de la contaminación del aire en la salud de la población: evidencias de estudios epidemiológicos. Salud Pública Mex. 2001;43:544-55.

4. Dockery DW, Pope III CA. Acute respiratory effects of particulate air pollution.. Annu Rev Public Health. 1994;15:107-32.

5. Gern JE. The Urban Environment and Childhood Asthma Study. J Allergy Clin Immunol. 2010;125:545-49.

6. Krieger J, Jacobs D, Ashley P, Baeder A, Chew G, Dearborn $D$, et al. Housing interventions and control of asthma-related indoor biologic agents: $A$ review of the evidence. J Public Health Manag Pract. 2010;16:S11-20.

7. Gavidia T, Pronczuk J, Sly P. Impactos ambientales sobre la salud respiratoria de los niños. Carga global de las enfermedades respiratorias pediátricas ligada al ambiente. Rev Chil Enf Respir. 2009;25:99-108.

8. Hernández A, Grineski S. Exploring the efficacy of an environmental health intervention in Ciudad Juárez, México. Fam Community Health. 2010;33:343-53.

9. Global Initiative for Asthma. GINA Report, Global Strategy for Asthma Management and Prevention. Fecha de consulta: 20 de abril del 2008. Disponible en: http://www.ginasthma. com/Guidelineitem.asp??|1=2\&/2=1\&intld=60.

10. U.S. Environmental Protection Agency (EPA). National air toxics program: The integrated urban strategy. Report to Congress. Fecha de consulta: 12 de enero del 2008. Disponible en: http://www.epa.gov/ttnatw01/urban/natprpt. pdf.

11. Bruce N, Pérez R, Albalak R. Indoor air pollution in developing countries: A major environmental and public health challenge. Bull World Health Organ. 2000;78:106771.

12. Hernández LA, Zárate A, Díaz LA. Factores de riesgo para infección respiratoria aguda con énfasis en la contaminación ambiental. Rev Salud UIS. 1993;21:7-15.

13. Dennis R, Caraballo L, García E, Cala LL, Caballero A, Aristizábal G, et al. Prevalencia de asma en seis ciudades de Colombia. Revista Colombiana de Neumología. 2000;13:485-93.

14. Agencia para Sustancias Tóxicas y el Registro de Enfermedades. Estudios de caso en medicina ambiental. Fecha de consulta: 15 de abril del 2008. Disponible en: www.atsdr.cdc.gov/es/csem/asma.

15. Salo PM, Xia J, Johnson CA, Li Y, Avol EL, Gong J, et al. Indoor allergens, asthma, and asthma-related symptoms among adolescents in Wuhan, China. Ann Epidemiol. 2004;14:543-50.

16. Lau S, Nickel R, Niggemann B, Grüber C, Sommerfeld C, Illi S, et al. The development of childhood asthma: Lessons from the German multicentre allergy study (MAS). Paediatr Rev Respir. 2002;3:265-72.

17. Salo PM, Arbes SJ Jr, Sever M, Jaramillo R, Cohn RD, London SJ, et al. Exposure to Alternaria alternata in US homes is associated with asthma symptoms. J Allergy Clin Immunol. 2006;118:892-8.

18. Ingibjörg M, Norbäck D, Björnsson E, Soon A, Jarvis D, Gislason D, et al. Indoor environment in three North European cities in relationship to atopy and respiratory symptoms. Clin Respir J. 2009;3:85-94.

19. Sun Y, Zhang Y, Sundell J, Fan Z, Bao I. Dampness in dorm rooms and its associations with allergy and airways infections among college students in China: A crosssectional study. Indoor Air. 2009;19:348-56.

20. Sharma HP, Hansel NN, Matsui E, Diette GB, Eggleston P, Breysse P. Influencias ambientales de interiores sobre el asma de los niños. Pediatr Clin North Am. 2007;54:103-20.

21. Rodríguez L, Rey JJ, Herrera A, Castro H, Niederbacher J, Bolívar F, et al. Prevalencia de síntomas respiratorios compatibles con asma y asociación con contaminación atmosférica en pre-escolares de Bucaramanga. Biomédica. 2010;30:15-22.

22. Dean J, Coulombier D, Smith D, Brendel K, Arner T, Dean A. Epi-info 6.04. Geneva: Word Health Organization; 1996.

23. Rodríguez L, Herrera A, Castro H, Niederbacher J, Vera L. Incidencia de síntomas respiratorios y su asociación con contaminación atmosférica en preescolares: un análisis multinivel. Cad Saude Pública. 2010;26:1411-8.

24. Chong HJ, Rosario N, De La Bianca AC, Solé D, Mallol J. Validation of questionnaire for epidemiologic studies of wheezing in infants. Pediatr Allergy Immunol. 2007;18:86-7.

25. Mata $\mathbf{C F}$, Fernández $\mathbf{M}$, Pérez $\mathbf{M}$, Guillén $\mathbf{F}$. Validation of the Spanish version of the phase III ISAAC questionnaire on asthma. J Invest Allergol Clin Immunol. 2005;15:201-10.

26. Orozco V. Medición en salud: diagnóstico y evaluación de resultados, un manual crítico más allá de lo básico. Bucaramanga: División de Publicaciones UIS; 2010.

27. Morgan W, Crain E, Gruchalla R, O'Connor G, Kattan M, Evans R, et al. Results of a home-based environmental intervention among urban children with asthma. $\mathrm{N}$ Engl $\mathrm{J}$ Med. 2004;351:1068-80.

28. Caballero R, Naber O, Morfin B. Correlación entre pruebas cutáneas positivas a hongos, lgE total e $\lg E$ específica por ELISA, y cultivos de hongos en el medio ambiente del paciente pediátrico alérgico. Rev Alergia Mex. 2001;5:137-40.

29. Cordero D, Soto M, Mejía M. Los nuevos patrones de crecimiento de la OMS. Ginebra: OPS/OMS; 2007. Fecha de consulta: 15 de octubre de 2007. Disponible en: http://www. ops.org.bo/textocompleto/naiepi-patrones-crecimiento.pdf.

30. StataCorp. Stata Statistical Software: Release 9. College Station, TX: Stata Corporation LP; 2007. 
31. Kleinbaum DG, Kupper LL, Muller KE, Nizan A. Applied regression analysis and other multivariable methods. Fourth edition. Belmont, CA: Thomson Higher Education; 2008.

32. Greenland S. Modeling and variable selection in epidemiologic analysis. Am J Public Health. 1989;79:340-9.

33. Vojta P, Friedman W, Marker D, Clickner R, Rogers J, Viet S, et al. First national survey of lead and allergens in housing: Survey design and methods for the allergen and endotoxin components. Environ Health Perspect. 2002;110:527-32.

34. Nilsson A, Kihlström E, Lagesson V, Wessén B, Szponar $\mathrm{B}$, Larsson L, et al. Microorganisms and volatile organic compounds in airborne dust from damp residences. Indoor Air. 2004;14:74-82.

35. Stark P, Celedón J, Ginger I, Ryan ML, Burge H, Muilenberg $\mathbf{M}$. Fungal Levels in the home and allergic rhinitis by 5 years of age. Environ Health Perspect. 2005;113:1405-9.

36. Antova T, Pattenden S, Brunekreef B, Heinrich J, Rudnai $\mathbf{P}$, Forastiere $\mathbf{F}$, et al. Exposure to indoor mould and children's respiratory health in the PATY study. J Epidemiol Community Health. 2008;62:708-14.

37. Storey E, Dangman K, Schenck, Debernardo R, Yang C, Bracker M, et al. Guidance for clinicians on the recognition and management of health effects related to mold exposure and moisture indoors. Fecha de consulta: 14 de septiembre de 2010. Disponible en:http://oehc.uchc.edu/clinser/ mold\%20guide.pdf.

38. Cruz A, Jiménez A. Evaluación de la contaminación del aire por microorganismos oportunistas y su relación con material particulado (PM 2.5 y PM 10) en la localidad de Puente Aranda, Bogotá, D.C. (trabajo de grado). Bogotá,.D.C.: Universidad de la Salle; 2006. p. 189-91.

39. Kim J, Elfman L, Norback D. Respiratory symptoms, asthma and allergen levels in schools comparison between Korea and Sweden. Indoor Air. 2007;17:122-9.

40. Miller JD. Fungi as contaminants in indoor air. Atmosph Environ. 1992;26:2163-72.

41. Pasanen AL. A review: Fungal exposure assessment in indoor environments. Indoor Air. 2001;11:87-9.

42. Garrett M, Rayment P, Hooper M, Abramson M, Hooper B. Indoor airbone fungal spores, house dampness and associations with environmental factors and respiratory health in children. Clin Exp Allergy. 1998;28:459-67.

43. Kowalski W J, Indoor mold growth. Fecha de consulta: 10 de septiembre de 2010. Disponible en: http://www.engr.psu. edu/ae/iec/abe/publications/Indoor_Mold_Growth.htm.

44. Pulimood T, Corden J, Bryden C, Sharples L, Nasser S. Epidemic asthma and the role of the fungal mold Alternaria alternate. J Allergy Clin Immunol. 2007;120:610-7.

45. Jeroen D, Pearce N. Invited commentary: Is indoor mold exposure a risk factor for asthma? Am J Epidemiol. 2003;158:203-6.

46. Varela Al. Correlación clínica y espirométrica con alergenos intradomiciliarios y con contaminantes. Alergia Méx. $2001 ; 48: 107$.
47. Chen CM, Gehring U, Wickman M, Hoek G, Giovannangelo $\mathbf{M}$, Nordling $\mathbf{E}$, et al. Domestic cat allergen and allergic sensitization in young children. Int $\mathrm{J}$ Hyg Environ Health. 2008;211:337-44.

48. Johnston R, Burge H, Fisk W, Gold D, Gordis L, Grunstein $\mathbf{M}$, et al. Clearing the air: Asthma and indoor air exposures is available for sale from the National. Washington, D.C.: Academy Press; 2000. Fecha de consulta: 18 de septiembre de 2009. Disponible en: http://www.nap.edu/openbook. php?isbn=0309064961.

49. Gaffin J, Phipatanakul W. The role of indoor allergens in the development of asthma. Curr Opin Allergy Clin Immunol. 2009;9:128-35.

50. Moreno L, Caraballo L, Puerta L. Importancia médica de los alérgenos de ácaros domésticos. Biomédica. 1995; 15:93-103.

51. De Alba J, Raemdonck K, Collins DA, Wong S, Nials A. House dust mite induces direct airway inflammation in vivo: Implications for future disease therapy? Eur Respir J. 2010;35:1377-87.

52. Oddy W, Pea J. Breastfeeding asthma, and atopic disease: An epidemiological review of the literature. J Hum Lact. 2003;19;250-61.

53. Ngoc P, Soto N, Ávila L, Hunninghake G, Raby B, Laskey $D$, et al. Paternal asthma, mold exposure, and increased airway responsiveness among children with asthma in Costa Rica. Chest. 2008;133:107-14.

54. Boulet L. Influence of comorbid conditions on asthma. Eur Respir J. 2009;33:897-6.

55. Porsbjerg C, von Linstow M, Ulrik C, Nepper S, Backer V. Risk factors for onset of asthma a 12-year prospective follow-up study. Chest. 2006;129:309-16.

56. Belanger K, Beckett W, Triche E, Bracken M, Holford T, Renp, et al. Persistent cough in the first year of life: Associations with indoor allergens, air contaminants, and maternal history asthma. Am J Epidemiol. 2003;158:195202.

57. Salo PM, Xia J, Johnson CA, Li Y, Avol EL, Gong J, et al. Indoor allergens, asthma, and asthma-related symptoms among adolescents in Wuhan, China. Ann Epidemiol. 2004;14:543-50.

58. Henriksen A, Holmen T, Bjermer L. Sensitization and exposure to petallergens in asthmatics versusnon asthmatics with allergic rhinitis. Respir Med. 2001;95:122:9.

59. Almqvist C, Egmar AC, Hedlin G, Lundqvist M, Nordvall SL, Pershagen G, et al. Direct and indirect exposure to pets risk of sensitization and asthma at 4 years in a bird cohort. Clin Exp Allergy. 2003;33:1190-7.

60. Takkouche B, González-Barcala FJ, Etminan M, Fitzgerald M. Exposure to furry pets and the risk of asthma and allergic rhinitis: A meta-analysis. Allergy. 2008;63:857-64.

61. Campo P, Kalra HK, Levin L, Reponen T, Olds R, Lummus ZL, et al. Influence of dog ownership and high endotoxin on wheezing and atopy during infancy. J Allergy Clin Immunol. 2006;118:1271-8.

62. García M, Mallol J, Dirceu S, Brand P. THE EISL STUDY GROUP. International study of wheezing in infants: Risk 
factors in affluent and non-affluent countries during the first year of life. Pediatric Allergy Immunol. 2010;21:878-88.

63. Lombardi E, Simoni M, La Grutta S, Viegi G, Bisanti L, Chellini E, et al. Effects of pet exposure in the first year of life on respiratory and allergic symptoms in 7-year-old children. The SIDRIA-2 study. Pediatric Allergy Immunol. 2010;21:268-76.

64. Hong SJ, Lee MS, Sohn MH, Shim JY, Han YS, Park KS, et al. Self-reported prevalence and risk factors of asthma among Korean adolescents: 5-year follow-up study, 19952000. Clin Exp Allergy. 2004;34:1556-62.

65. Tsai C, Huang J, Hwang BJ, Lee Y. Household environmental tobacco smoke and risks of asthma, wheeze and bronchitic symptoms among children in Taiwan. Respir Res. 2010;11:1-11.

66. Antova T, Pattenden S, Nikiforov B, Leonardi GS, Boeva B, Fletcher $\mathbf{T}$, et al. Nutrition and respiratory health in children in six central and Eastern European countries. Thorax. 2003;58:231-6.

67. Chiarella P. Prevalencia de síntomas respiratorios compatibles con asma en niños escolares de 13 y 14 años de San Martín de Porres (Lima). Enferm Tórax. 2004;48:50-8.
68. Lau S, LIli S, Sommerfeld C, Niggemann B, Mutius E, Wahn U. Early exposure to house dust mite and cat allergens and development of childhood asthma: A cohort study. Lancet. 2000;356:1392-7.

69. Schumacker R. Reliability a Rasch perspective. Educ Psychol Meas. 2007;67:394-409.

70. Deddens JA, Petersen MR. Approaches for estimating prevalence ratios. Occup Environ Med. 2008;65:501-6.

71. Zocchetti C, Consonni D, Bertazzi PA. Relationship between prevalence rate ratios and odds ratios in cross sectional studies. Int J Epidemiol. 1997;26:220-3.

72. Caraballo L. Factores de riesgo en asma. Acta Med Colomb. 2006;31:91-4

73. Sheikh A, Hurwitz B, Shehata Y. Medidas para evitar los ácaros del polvo doméstico para la rinitis alérgica perenne. Cochrane Plus. 2008, número 2. Fecha de consulta: 26 de septiembre de 2010. Disponible en: http://www.updatesoftware.com/pdf/CD001563.pdf. 